\title{
Experimental Investigation of Changing Fuel Path Dynamics in Twin-Independent Variable Camshaft Timing Engines
}

\author{
Umut Genç, Richard Ford, Keith Glover and Nick Collings \\ University of Cambridge
}

Copyright (@ 2001 Society of Automotive Engineers, Inc.

\begin{abstract}
The effect of a variable camshaft timing (VCT) disturbance on air-to-fuel ratio (AFR) signal is investigated for a twin-independent (TI) VCT engine. Different types of VCT disturbances on AFR signal are investigated. Gaseous fuel experiments are performed in addition to conventional petrol fuel experiments to show that not all the transient VCT disturbances acting on AFR are due to changes in air path dynamics. Experiments show that varying exhaust valve closing (EVC) timing has a significant effect on the air path dynamics and is an important cause of transient AFR deviations. However varying EVC does not affect the fuel puddle significantly. On the other hand varying inlet valve opening (IVO) timing has a strong effect on the fuel puddle size and is also an important cause of transient AFR deviations. Thus for superior transient AFR control in TI-VCT engines, it is essential to model not only the effects of valve timings on air path dynamics but also on the fuel path dynamics.
\end{abstract}

\section{INTRODUCTION}

Variable valve timing (VVT) mechanisms have the potential to improve fuel economy, performance and emission levels of gasoline engines [3]. The particular VVT mechanism investigated in this paper is variable camshaft timing. It changes the phase of the camshafts relative to the crankshaft without changing the valve profile itself. The most effective VCT system is the TIVCT, which can vary the phase of the intake and exhaust cams independently. It has the ability not only retard/advance valve events but also vary the valve overlap.

Several benefits of the TI-VCT engines are reported in the literature [7]. Most of the emission improvements are due to the internal exhaust gas recirculation (EGR) mechanism realisable by VCT. The effective use of internal EGR can reduce both $\mathrm{NO}_{\mathrm{x}}$ and $\mathrm{HC}$ emissions. In addition higher levels of residual gas in the cylinders means higher values of manifold pressure for a given load. This has the effect of reducing pumping losses and therefore improves fuel economy. Furthermore, TI-VCT permits the optimisation of the valve timing over a wide range of engine operating conditions, allowing both improved idle quality (requiring little or no overlap) and optimal breathing at high speed/load (large overlap).

In order to get most out of VCT, it is essential to change the valve timings in an aggressive way during the engine operation. For example, for adequate torque control during tip-ins and tip-outs, the speed of the VCT response should match the speed of the manifold filling and emptying dynamics [7]. This can be achieved by precise and fast control of the VCT actuators. The identification and control of VCT actuators is in itself an interesting problem and a detailed analysis can be found in [1]. Sudden changes in VCT disturb both engine torque and AFR since it alters the engine breathing characteristics. The torque disturbance may cause driveability problems and therefore is undesirable. On the other hand, AFR disturbances will reduce the catalyst conversion efficiency. This may significantly degrade the emission benefits of VCT engines. In particular the transient effects of the VCT disturbances are much more grave and require active compensation to recover the full benefits of VCT mechanism [6].

In the following the transient AFR control problem in TIVCT engines is investigated with a focus on fuel path dynamics. The main question for this study is whether the system changes caused by rapidly varying the cam timing have a significant effect on the fuel puddle dynamics in VCT engines. First, a short description of the transient AFR control problem is given. Then experimental observations of the transient VCT disturbance on AFR are reported. Next gaseous fuel experiments are presented as evidence that not only air dynamics but also fuel path dynamics is affected by VCT. Finally, the future directions for superior AFR control in VCT engines are outlined.

The engine used in the experiments is a 4-cylinder 1.6 litre PFI TI-VCT engine. IVO angle can vary between -30 
and 15 degrees after top dead centre (ATDC). Similarly, EVC angle can vary between -15 and 30 degrees ATDC.

\section{THE AFR CONTROL PROBLEM}

Figure 1 shows a simplified control oriented model of the AFR loop for a TI-VCT engine. The model output is defined in terms of lambda (normalised AFR). There are two important dynamics here: air path dynamics and the fuel path dynamics. Air path dynamics are composed of throttle, manifold pressure $\left(P_{m}\right)$ and cylinder mass airflow $\left(m_{a c}\right)$. Although the figure does not show it explicitly, manifold pressure is a function of both throttle position (TP) and cylinder airflow. It is known that the cylinder airflow is a function of manifold pressure and valve timings (IVO and EVC). This is how the VCT disturbs the air path mainly, i.e. by altering the cylinder airflow rate.

The fuel path is composed of injection delay, wallwetting, cycle and transport delay, gas mixing and the AFR sensor dynamics. The injection delay occurs due to the fact that the fuel is injected onto closed valves before IVO. Wall-wetting dynamics describes the fact that not all of the injected fuel $\left(\mathrm{m}_{\mathrm{fi}}\right)$ is sucked into the cylinder immediately. Some of the fuel impinges onto the inlet port wall and enters the cylinder gradually through evaporation. Usually a simple first order fuel puddle model is used to describe this process for control purposes. The normalised AFR is defined as the ratio of the cylinder airflow rate to cylinder fuel flow rate $\left(\mathrm{m}_{\mathrm{fc}}\right)$ as

$$
\lambda_{c}=\frac{m_{a c}}{m_{f_{c}}}
$$

The normalisation constant is not shown explicitly in equation (1). Cycle and transport delays are the main delays in the loop. Cycle delay is around two events (compression and expansion strokes). Transport delay varies from one event to several events depending on the engine load. Gas mixing occurs in the exhaust manifold before the lambda sensor, which can have a significant effect especially if the sensor is placed further away from the exhaust valves. The final dynamics in the fuel path is the sensor, which is usually described by either a first or a second order model. The sensor output, exhaust lambda, is denoted as $\lambda_{x}$.

There have been several publications in the literature proposing feedback and feedforward control schemes for transient AFR control in engines with VCT mechanisms $[5,4,2]$. In all of these publications the mean value engine models used for control include only the air path dynamics and the effects of VCT on the cylinder air charge. On the other hand, it is known that the fuel path dynamics varies with engine load, wall temperature and mixture backflow [8]. Therefore, it is likely that the fuel path dynamics will also be affected by changing valve timings.

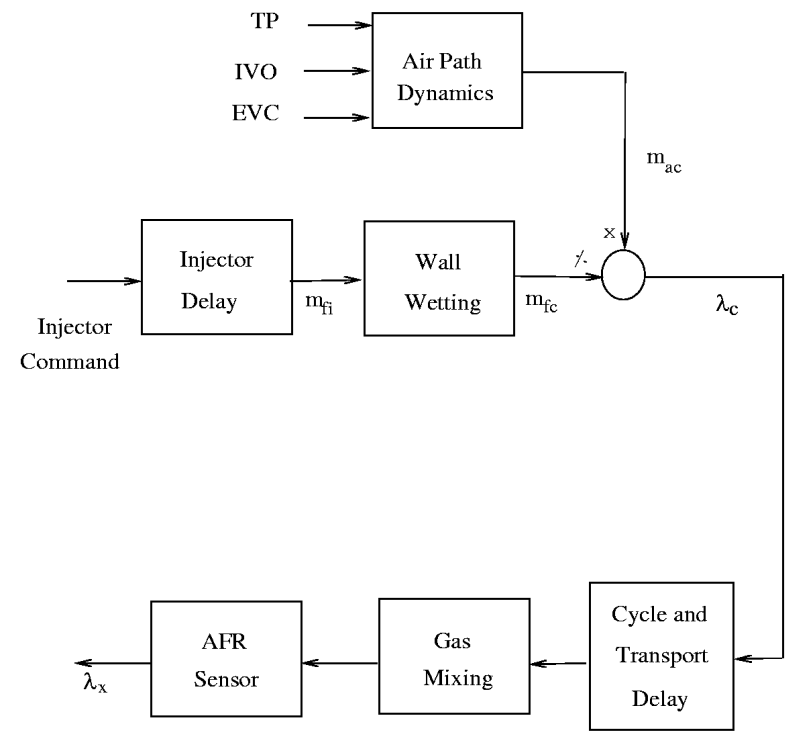

Figure 1. Control oriented model of the AFR loop. Although effects of the IVO and EVC on the air dynamics are known, their effects on the fuel path dynamics are not well defined so far.

\section{TRANSIENT VCT DISTURBANCES ON AFR}

Effects of the IVO and EVC timings on the AFR are investigated separately through engine tests. The experiments are performed at $1500 \mathrm{rpm}$ with constant throttle angle and fuel injection. A square wave reference is applied to the valve timings and the results are averaged to obtain a representative response. Sampling is every 180 degrees (1 event). The AFR signal is measured at the exhaust of cylinder one by a wide-range lambda sensor.

Figure 2 shows a transient disturbance on the lambda signal when the overlap is varied between $0^{\circ}$ and $30^{\circ}$ ATDC by exciting EVC (IVO is fixed at $-5^{\circ}$ ATDC). The figure also shows the throttle mass airflow $\left(\mathrm{m}_{\mathrm{at}}\right)$, and the manifold pressure traces. When the overlap increased by late EVC, the volumetric efficiency is reduced (evident from the $m_{a t}$ trace). This causes a rich spike in the lambda reading at around 0.5 seconds. And the increase in manifold pressure is due to the fact that cylinder airflow is quicker to respond to VCT than the throttle airflow. Hence there is a temporary period when less air is sucked into the cylinder, which causes the rich spike in lambda, than the air entering into the manifold via throttle. This results in an increase in the manifold pressure. Similarly, when overlap is reduced by advancing EVC, a lean spike is observed at around 5.5 seconds. The cause of the lean spike can be explained using the same arguments. It is interesting that the transient EVC disturbance on AFR (Figure 2) can be explained by only analysing the changes in the air path dynamics due to varying EVC. 

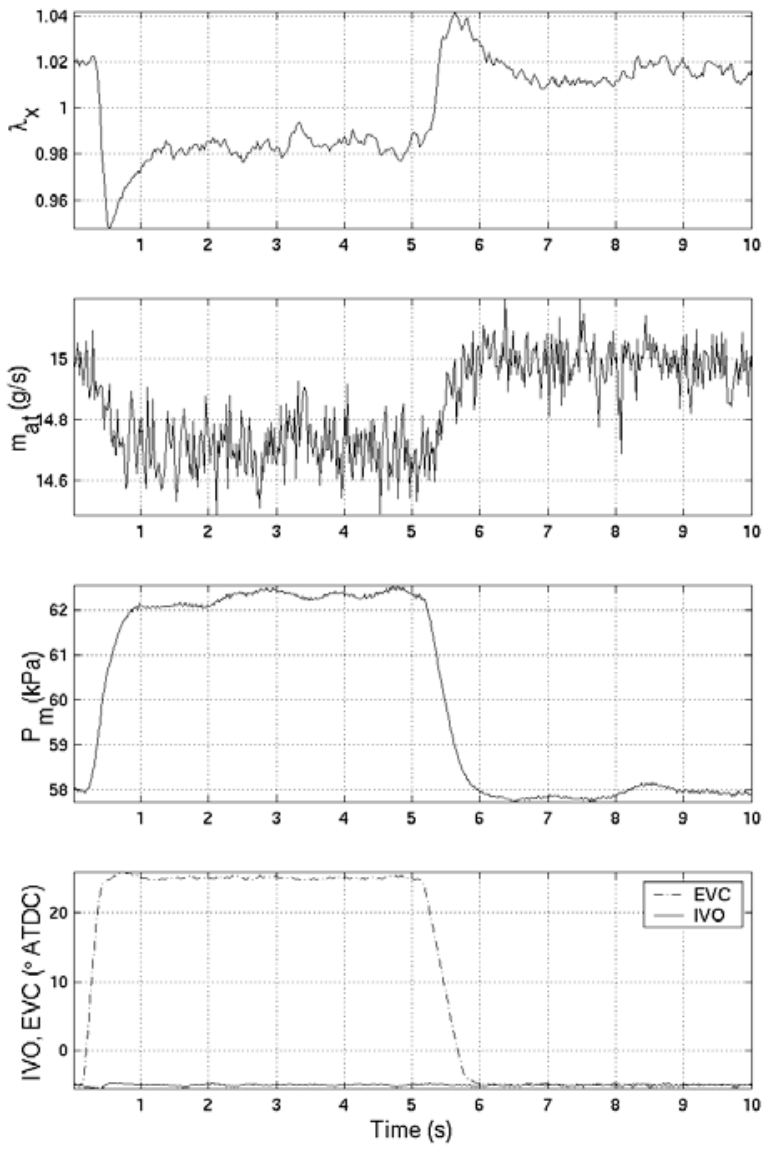

Figure 2. Transient EVC disturbance on the AFR signal

The same experiment is performed this time by varying the overlap via control of IVO while keeping EVC fixed at $10^{\circ}$ ATDC. The mean traces are shown in Figure 3. Extending the overlap into the exhaust stroke increases the volumetric efficiency, which is evident from the slight increase in throttle airflow. Thus it is expected that there should be a lean spike in lambda at around 0.5 seconds if one is to use similar arguments to the EVC disturbance case. However, instead there is a rich spike in the measurement. A similar unexpected observation happens when the overlap is reduced to zero by retarding IVO, i.e. a lean spike in lambda at around 5.5 seconds. On the other hand manifold pressure trace supports the throttle airflow behaviour. When throttle airflow increases, manifold pressure decreases as in the case of EVC disturbance and vice versa. Yet the lambda trace has its spikes in opposing directions to what would be expected from the air dynamics. This suggests that there is some extra dynamic effect in the measurements, which is related to neither throttle airflow nor manifold pressure.
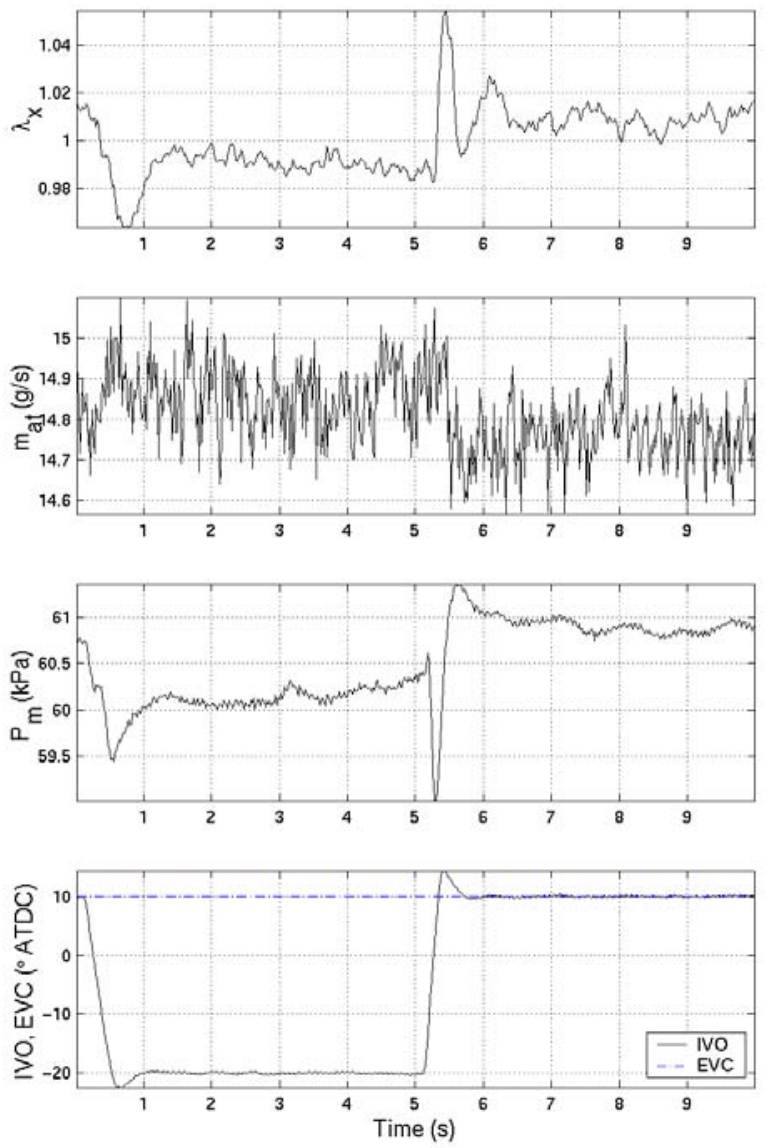

Figure 3. Transient IVO disturbance on the AFR signal

These experimental results show that not all the transient VCT disturbances on the AFR signal can be explained by the change in air path dynamics only. In particular, although EVC disturbance on AFR can be explained by changing air path dynamics, the same cannot be argued for the IVO disturbance on AFR.

Next, in order to get a better insight, the same experiments are repeated with gaseous fuel injection into the inlet port rather than the conventional petrol fuel.

\section{GASEOUS FUEL EXPERIMENTS}

The same experiments are performed with propane as the fuel rather than the conventional petrol. The propane is injected into the inlet port of cylinder one only. Since the propane is gaseous, the AFR readings are independent of the wall-wetting dynamics. This can be seen from the definition of the lambda for gaseous fuel operation, 


$$
\lambda_{c}^{g}=\frac{m_{a c}}{m_{f i}}
$$

as $m_{\mathrm{fi}}$ is constant during the experiments, any variations in the lambda trace are due to the variations in cylinder airflow rate for the gaseous fuel operation.
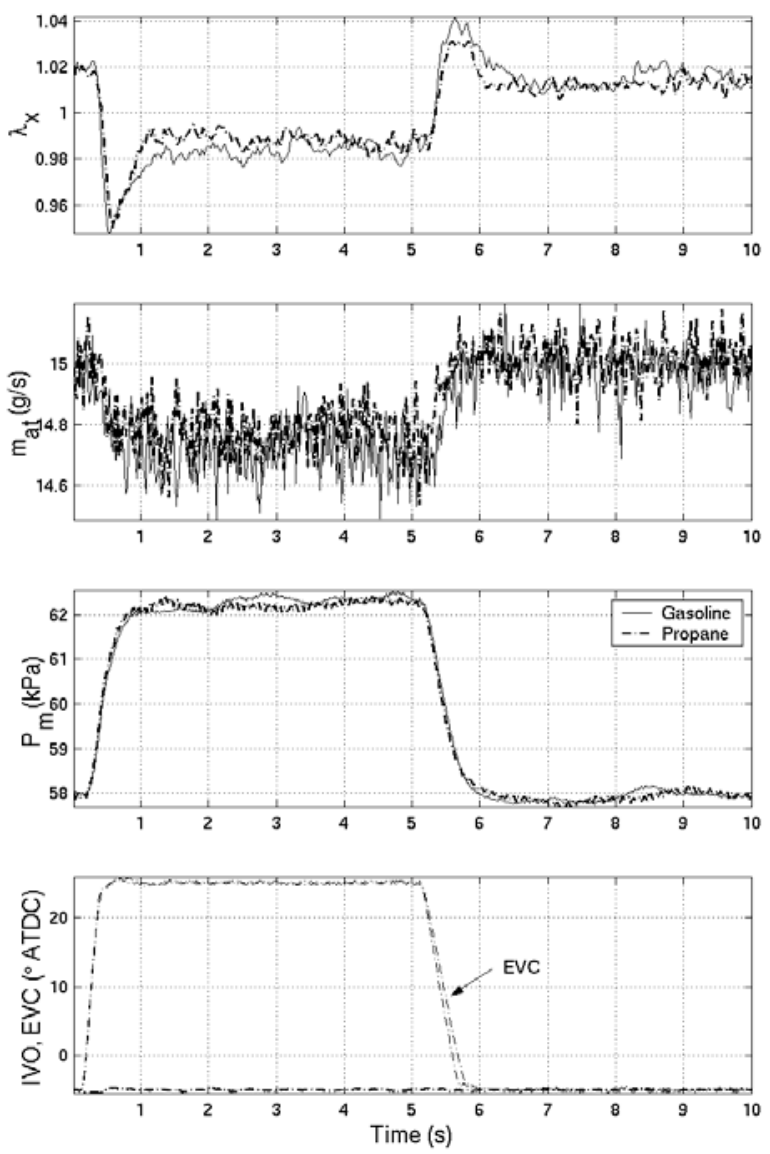

Figure 4. Transient EVC disturbance on the AFR signal: comparison of the petrol fuel and the gaseous fuel experiments.

Figure 4 depicts both the gaseous fuel and the petrol fuel (shown in Figure 2) experiments for the same variation in EVC. Very small difference between the two lambda readings show that this transient is caused only by the variation in the cylinder air flow. Moreover, such a small difference is only possible if there is no significant change in the wall-wetting dynamics under this particular EVC disturbance.

The next plot in Figure 5 shows the gaseous fuel experiment for the IVO disturbance on AFR together with the previous petrol fuel result (shown in Figure 3 ). The gaseous fuel lambda reading is just what is expected from changing air path dynamics. The increase in overlap by advancing IVO improves the volumetric efficiency as is evident from the throttle airflow trace. This in turn causes a lean spike in lambda trace at around 0.5 seconds as in the case of EVC disturbance. Similarly a rich spike is observed when the overlap is reduced at around 5.5 seconds. Therefore, for gaseous fuel experiments all the transients VCT disturbances (varying EVC or IVO) on AFR can be explained by changes in the air path dynamics only. This is an expected result and indeed confirms that there is no wallwetting dynamics for gaseous fuel operation.
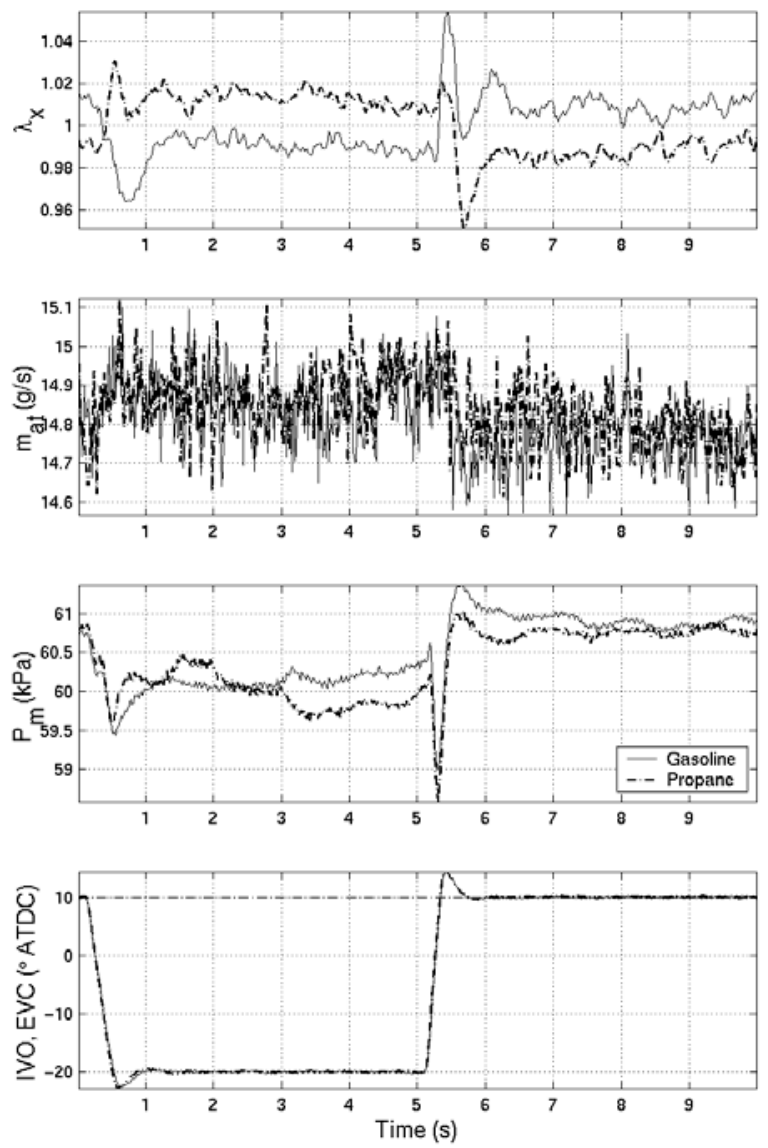

Figure 5. Transient IVO disturbance on the AFR signal: comparison of the petrol fuel and the gaseous fuel experiments.

On the other hand the results of the IVO disturbance on AFR with petrol fuel can only be explained by a significant change in the fuel path dynamics. In particular, a rich spike in the lambda trace (solid line in Figure 5) while the cylinder airflow is going through a lean spike (dashed-dotted line in Figure 5) is only possible with a big increase in the mass of fuel entering into cylinder. Since injected fuel is constant during the experiments, this extra fuel can only be coming from the fuel puddle itself. 
Fuel flow rate out of the fuel puddle during the IVO disturbance experiments can be estimated by taking ratio of the gaseous fuel lambda trace to petrol fuel lambda trace. Using equations (1) and (2), one get the estimate as

$\hat{m}_{f p}=\frac{m_{f c}}{m_{f i}}$

Since the injected fuel, $\mathrm{m}_{\mathrm{fi}}$, is constant, any variation in the estimated value is due to changes in the fuel puddle size.
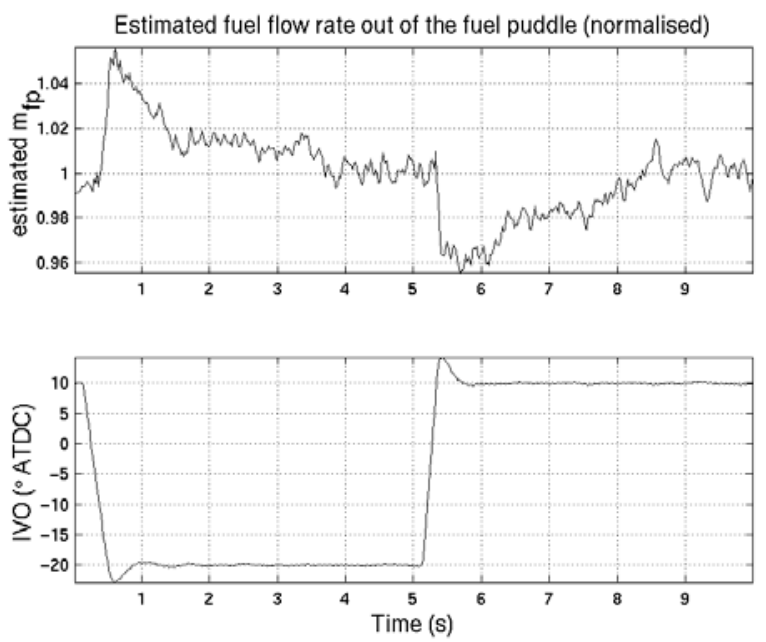

Figure 6. Estimated fuel flow rate out of the fuel puddle under IVO disturbance

The estimated fuel flow rate out of the fuel puddle, $\hat{m}_{f p}$, is plotted in Figure 6 together with the corresponding IVO trace. The plot shows that $\hat{m}_{f p}$ increases temporarily due to a decrease in fuel puddle mass, when the overlap is increased by advancing IVO at around 0.5 seconds. Similarly, when the overlap is reduced to zero at around 5.5 seconds, $\hat{m}_{f p}$ decreases temporarily due to an increase in fuel puddle mass.

It is evident that varying the overlap by altering IVO has a significant effect on the fuel puddle whereas varying it by altering EVC has a negligible one. This difference cannot be explained by changes in either throttle airflow or manifold pressure since they both vary with IVO and EVC. A possible explanation is given below.

During the experiments the inlet port temperature of cylinder one is also measured, which is depicted in Figure 7. The thermocouple time constant is of the order of 3 seconds. Both EVC and IVO variations change the port temperature. In particular increasing valve overlap increases the inlet port temperature since there is more backflow of residual gases into the inlet port and manifold. However, it is observed that early IVO causes a much bigger change in the port temperature than the late EVC for the same overlap length (especially at low loads). This may be because with early IVO, the piston is moving upwards for the most of the overlap period and is pushing residual gasses into the intake manifold. On the other hand with late EVC, piston is moving downwards for the most of the overlap and therefore pulling residual gasses into the cylinder. Such a difference in amount of residual gas sucked back into the inlet port may explain the much smaller increase in the temperature caused by retarding EVC. And the smaller variation in port temperature may explain why fuel puddle size does not change significantly. Note that the actual change of the port temperature is probably much faster than the measurements presented due to the slow dynamics of the thermocouple.

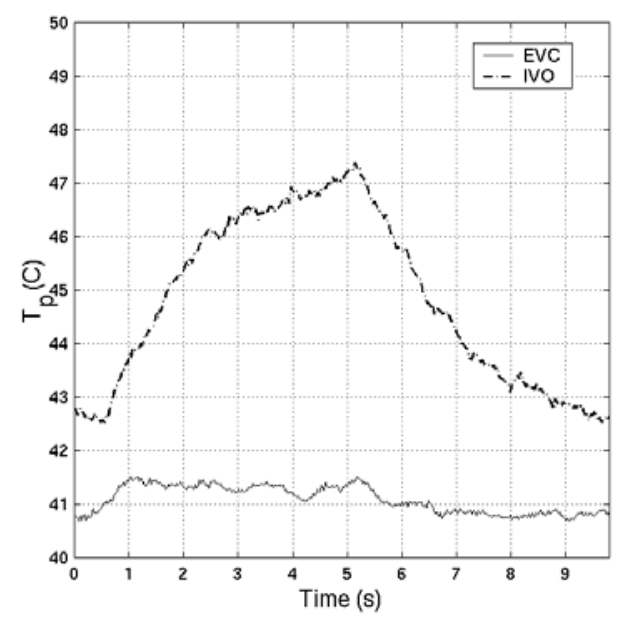

Figure 7. Measured inlet port temperature for EVC and IVO excitations at $60 \mathrm{kPa}$.

Results also suggest that even a perfect cylinder air flow predictor can not achieve a tight transient AFR control in a TI-VCT engine due to up to $5 \%$ change in the fuel flow rate out of the fuel puddle during transients. Such a change in fuel flow cannot be compensated for without a fuel flow predictor, which must capture the variation of fuel puddle parameters with valve timings.

\section{CONCLUSION}

Experimental results showing that variations in valve timings through a TI-VCT mechanism not only change the air path dynamics but also fuel path dynamics in a TIVCT engine are presented. Cylinder airflow rate is affected by variations in both EVC and IVO although EVC has a stronger effect. On the other hand IVO has a strong effect on the wall-wetting dynamics in the inlet port. In particular it is observed that advancing IVO reduces the fuel puddle size and retarding IVO increases it. Whereas varying EVC does not change the fuel puddle size significantly. 
We also show that for superior AFR transient compensation it is essential to take into account the dynamic effects of VCT on the fuel path especially on the fuel puddle. Next step should be developing sound methods to identify a parametric model that can capture the changes in wall-wetting, gas mixing and sensor dynamics with respect to variations in VCT.

\section{ACKNOWLEDGMENTS}

Umut Genç is funded by the Ford Motor Company. The engine research facilities in Cambridge University Engineering Department are financed jointly by the Ford Motor Company and Engineering and Physical Sciences Research Council.

\section{REFERENCES}

1. A.U. Genç, K.Glover, R. Ford; Nonlinear Control of Hydraulic Actuators in Variable Cam timing Engines. In MECA International Workshop, University of Salerno, Italy, September 2001.

2. D. Gorinevsky, J. Cook, L. Feldkamp, G. Vukovich; Predictive Design of Linear Feedback/Feedforward Controller for Automotive VCT Engines. In ACC 99, volume 1, pages 207-211, June 1999.
3. C. Gray. A Review of Variable Engine Valve Timing. SAE, (880386), 1988.

4. C.S. Hsieh, S.J. Freudenberg, and A.G. Stefanopoulou; Multivariable Controller Structure in a Variable Cam Timing Engine with Electronic Throttle and Torque Feedback. In Conf. on Control Appl., pages 465-470, Hawai, 1999.

5. S.C. Hsieh, A.G. Stefanopoulou, J.S. Freudenberg, and K.R. Butts. Emissions and Drivability Tradeoffs in a Variable Cam Timing SI Engine with Electronic Throttle. In ACC, pages 284-288, Albuquerque, New Mexico, June 1997.

6. M. Jankovic, F. Frischmuth, A.G. Stefanopoulou, and J.A. Cook; Torque Management of Engines with Variable Cam Timing. IEEE Control Systems, 18(5): 34, 1998.

7. T.G. Leone, E.J. Christenson, and R.A. Stein. Comparison of Variable Camshaft Timing Strategies at Part Load. SAE, (960584), 1996.

8. R.C. Turin, E.G.B. Casartelli, and H.P. Geering. A New Model for Fuel Supply Dynamics in an SI Engine. SAE, (940208), 1994. 